\title{
Differences in Leaf Color and Stage of Development at Harvest Influenced Phytochemical Content in Three Cultivars of Kale (Brassica oleracea L. and B. napus)
}

\author{
Nicole L. Waterland ${ }^{1}$, Youyoun Moon ${ }^{1}$, Janet C. Tou ${ }^{2}$, Dean A. Kopsell ${ }^{3}$, Moo Jung Kim ${ }^{1,2} \&$ Suejin Park ${ }^{1}$ \\ ${ }^{1}$ Division of Plant and Soil Sciences, West Virginia University, Morgantown, WV, USA \\ ${ }^{2}$ Division of Animal and Nutritional Sciences, West Virginia University, Morgantown, WV, USA \\ ${ }^{3}$ Department of Plant Sciences, The University of Florida, Gainsville, FL, USA \\ Correspondence: Nicole L. Waterland, Division of Plant and Soil Sciences, West Virginia University, \\ Morgantown, WV, 26506, USA. Tel: 1-304-293-2969. E-mail: nicole.waterland@mail.wvu.edu
}

Received: November 17, 2018

Accepted: December 20, 2018

Online Published: February 15, 2019

doi:10.5539/jas.v11n3p14

URL: https://doi.org/10.5539/jas.v11n3p14

The research is supported by the West Virginia University Foundation and the National Institute of Food and Agriculture, U.S. Department of Agriculture, HATCH accession numbers 1001653 and 228807.

\begin{abstract}
Microgreens and red colored plants have been suggested to contain higher level of health promoting phytochemicals. Kale (Brassica oleracea L. and other species) is regarded as a 'superfood' due to its antioxidant properties. In our study, three kale cultivars (B. oleracea L. 'Dwarf Blue Curled' and 'Scarlet', and B. napus 'Red Russian') differing in leaf pigmentation were harvested at five different leaf developmental stages and the concentrations of bioactive antioxidants compounds were evaluated. Carotenoids were measured by high performance liquid chromatography, and spectrometry analyses were used for total phenolics and anthocyanin measurements. Red leaf kale ('Scarlet') was generally higher in total carotenoids, phenolics, and anthocyanins than green leaf kales ('Dwarf Blue Curled' and 'Red Russian'). As kale matured, water content decreased and dry mass increased. On a dry weight basis, total carotenoids were more abundant in microgreens and young seedlings with 4 to 6 true leaves (baby greens) than adult stage. In contrast, on a fresh weight basis, baby greens or adult kales generally contained more carotenoids and total phenolic compounds than microgreens, more likely due to the lower dry mass and phytochemical accumulation at microgreen stages. Although some microgreens vegetables may contain more health promoting phytochemicals, based on our study, higher phytochemicals were detected in young seedlings or mature leaves of kale.
\end{abstract}

Keywords: anthocyanins, carotenoids, kale, leaf development, microgreens, phenolics

\section{Introduction}

Brassicas, the most widely consumed plants in the world, can provide a significant source of nutrients and bioactive compounds to the daily diet (Kapusta-Duch, Kopec, Piatkowska, Borczak, \& Leszczynska, 2012). Epidemiological studies have shown that Brassica vegetables reduce risk of cardiovascular disease and certain cancers, specifically gastric cancer(s) (Rodríguez-Cantú et al., 2011; Wu, Yang, Wang, Han, \& Xiang, 2013; Pagliaro, Santolamazza, Simonelli, \& Rubattu, 2015). The health promoting properties of Brassica vegetables have been attributed to bioactive compounds with antioxidant and free radical scavenging properties (Ismail, Marjan, \& Foong, 2004; Podsędek, 2007; Singh, Upadhyay, Prasad, Bahadur, \& Rai, 2007). Kale (Brassica oleracea $\mathrm{L}$. and other species), which consists of mainly B. oleracea cultivars and some cultivars of $B$. napus, has been reported to have the highest antioxidant capacity among Brassica vegetables (Podsędek, 2007). This may be due to kale being a rich source of carotenoids with the highest xanthophyll (lutein, zeaxanthin) content among leafy green vegetables (Perry, Rasmussen, \& Johnson, 2009). However, carotenoid content in kale can vary depending on factors such as: cultivation practices (e.g. greenhouse, field, hydroponics), cultivars of different leaf color, and maturity at harvest (Walsh, Bartlett, \& Eperjesi, 2015). To our knowledge, no research 
has been conducted on any crop grown under the same environmental conditions to examine the effect of the leaf development stages on the phytochemical content.

There has been an increasing interest in consumption of red vegetables and fruits for their antioxidant pigments. Carotenoids are present in many fruits and vegetables displaying red, orange or yellow coloration (Bartley \& Scolnik, 1995). Anthocyanins, phenolic compounds, present in leaves, flowers and fruits displaying reddish, bluish, and purple hues (He \& Giusti, 2010). Olsen, Aaby, and Borge (2010) reported higher anthocyanins, but lower total phenolics in red compared to green curly kale. During leaf development, pigmentation can also change (Lefsrud, Kopsell, Wenzel, \& Sheehan, 2007). Timing of harvest is of interest with the emerging popularity of immature leafy greens such as microgreens (cotyledons with or without two fully expanded true leaves) and baby leaf (young seedlings with 4 to 6 true leaves) due to suggestions of higher concentrations of bioactive compounds than mature plants. Xiao, Lester, Luo, and Wang (2012) examined nutritional contents of 25 commercially available fresh microgreens and found that several microgreens contain higher levels of phytochemicals than mature leaves.

Therefore, the objectives of this study were to investigate possible genetic and physiological impacts among kale cultivars with different leaf color and across different developmental stages on carotenoids, total phenolics, and anthocyanin concentrations. A better understanding of the influence of cultivar and developmental stage on these antioxidant compounds can assist growers and consumers in selecting the kale with a greater health-promoting values.

\section{Materials and Methods}

\subsection{Plant Materials and Growing Conditions}

Three kale cultivars were selected for their different leaf pigmentation, green leaf 'Dwarf Blue Curled' (DBC, $B$. oleracea L.), red leaf 'Scarlet' (B. oleracea L.) and green leaf with purple veins 'Red Russian' (RR, B. napus). All kale cultivars were grown in the peat moss based soilless media (Sunshine Mix \#1, Sun Gro Horticulture, Agawam, MA) in a greenhouse (Morgantown, WV) under natural irradiance from June 6 to Sept 8, 2015. Mean greenhouse temperatures were $24.4 / 19.7 \pm 3.7 / 2.2{ }^{\circ} \mathrm{C}$ day/night (mean $\pm \mathrm{SD}$ ). Kale were fertigated with a $25 \%$ Hoagland modified nutrient solution \#2 (PhytoTechnology Lab., Shawnee Mission, KS). After harvested, plants were freeze-dried (VirTis Freezemobile 12SL, SP Scientific, Warminster, PA) and ground to powder, and then stored at $-80{ }^{\circ} \mathrm{C}$ until analyses.

Kale was grown to five defined developmental stages, fully expanded cotyledon (microgreen 1 or MG1), seedlings with two true leaves (microgreen 2 or MG2), seedlings with four true leaves (baby leaf 1 or BL1), seedlings with six true leaves (baby leaf 2 or BL2) and plants with more than eight true leaves (adult). Leaves of 'DBC' and 'RR' were collected on 8, 13, 18 and 37 days after seeds were sown for MG1, MG2, BL1 and BL2 stages, respectively, while it required 9, 16, 29, 44 days for 'Scarlet' to be harvested for each corresponding stage. For adult stage, all kales were harvested 89 days after seeds were sown. All aerial plant tissues were collected for sample preparation.

\subsection{Carotenoid Analysis}

Carotenoids were analyzed according to Kopsell, Barickman, Sams, \& McElroy (2007). Briefly, freeze-dried samples $(0.1 \mathrm{~g})$ were re-hydrated in $0.8 \mathrm{~mL}$ of ultrapure water for $10 \mathrm{~min}$. Ethyl- $\beta-8$ '-apo-carotenoate (Carotenature, Lupsingen, Switzerland) was added as an internal standard $(0.8 \mathrm{~mL})$. Tetrahydrofuran (THF)(2.5 $\mathrm{mL}$ ) was stabilized with 2,6-di-tert-butyl-4-methoxyphenol $\left(25 \mathrm{mg} \mathrm{L}^{-1}\right)$. Samples were homogenized prior to centrifuging at $500 g_{\mathrm{n}}$ for $3 \mathrm{~min}$. The supernatant was collected and samples were re-extracted in $2 \mathrm{~mL}$ of THF as described above. The combined supernatants were evaporated under a stream of nitrogen to $0.5 \mathrm{~mL}$, then reconstituted to a final volume of $5 \mathrm{~mL}$ with acetone. Final extracts of $2 \mathrm{~mL}$ were filtered through a $0.2-\mu \mathrm{m}$ polytetrafluoroethylene filter (Model Econofilter PTFE 25/20, Agilent Technologies, Wilmington, DE). A high performance liquid chromatography (Agilent 1200 series, Agilent Technologies, Palo Alto, CA) equipped with a photodiode array detector was used for carotenoid analysis. A 250 x $4.6 \mathrm{~mm}$ i.d., $5 \mu \mathrm{m}$ analytical scale polymeric $\mathrm{C}_{30}$ reverse-phase column (ProntoSIL, MAC-MOD Analytical Inc., Chadds Ford, PA) was used. Carotenoids and internal standard in the eluents from a $20 \mu \mathrm{L}$ injection loop were detected at $453 \mathrm{~nm}$. Carotenoids identified were lutein, $\beta$-carotene, neoxanthin, violaxanthin, antheraxanthin, $\alpha$-carotene and zeaxanthin. Individual carotenoids were quantified using an external standard (ChromaDex Inc., Irvine, CA).

\subsection{Total Phenolic Compounds and Anthocyanin Analyses}

Total phenolic compounds and anthocyanin were extracted using the method of Nicolle et al. (2004) and the total phenolic and anthocyanin contents were analyzed following the method of Olsen et al. (2010). Absorbance was 
measured at 520 and $700 \mathrm{~nm}$ using a spectrophotometer (Spectronic ${ }^{\circledR}$ Genesys $^{\mathrm{TM}} 5$, Thermo Fisher Scientific Inc., Waltham, MA).

To determine the total phenolic content, $0.3 \mathrm{~mL}$ of extract or gallic acid standard was mixed with $1.5 \mathrm{~mL}$ of 0.5 $\mathrm{N}$ Folin-Ciocalteu reagent (Sigma-Aldrich, St. Louis, MO) and the mixture was incubated for $5 \mathrm{~min}$, then $1.5 \mathrm{~mL}$ of sodium carbonate solution $(0.5 \mathrm{M})$ was added. The mixture was incubated for $2 \mathrm{~h}$ at room temperature in the dark before measuring absorbance at $765 \mathrm{~nm}$ using a spectrophotometer (Spectronic ${ }^{\circledR}$ Genesys $^{\mathrm{TM}}$ 5, Thermo Fisher Scientific Inc., Waltham, MA). The total phenolic content was determined using a gallic acid standard curve $\left(\mathrm{y}=0.1366 \mathrm{x}+0.0054, \mathrm{R}^{2}=0.9988\right)$ and expressed as gallic acid equivalent. Total anthocyanins were calculated according to the following equation and the total anthocyanin content was expressed as cyanidin 3 -glucoside equivalent.

$$
\text { Total Anthocyanins }\left(\frac{\mathrm{mg}}{\mathrm{L}}\right)=\frac{\mathrm{A} \times \mathrm{MW} \times \mathrm{DF} \times 1000}{\varepsilon \times 1}
$$

Where, $\mathrm{A}=\left(\mathrm{A}_{520}-\mathrm{A}_{700}\right) \mathrm{pH} 1.0-\left(\mathrm{A}_{520}-\mathrm{A}_{700}\right) \mathrm{pH} 4.5 ; \mathrm{MW}=$ Molecular Weight for cyanindin-3-glucoside (449.2 $\left.\mathrm{g} \mathrm{mol}^{-1}\right) ; \mathrm{DF}=$ Dilution Factor; $\varepsilon=$ molar extinction coefficient $(26,900) ; 1=$ path length $(1 \mathrm{~cm})$.

The concentrations of carotenoids and total phenolics were calculated on a fresh weight $(\mathrm{FW})$ and dry weight (DW) basis. Dry mass of kale leaves was determined in percentage by comparing DW (freeze-dried) with FW at each developmental stage.

\subsection{Statistical Analysis}

Experiments were conducted as a randomized complete block design with four replications $(n=4)$. Analysis of variance (ANOVA) was performed by SAS version 9.2 (SAS Institute, Inc., Cary, NC). Kale cultivars were blocked by replication based on plant position in the greenhouse. Differences among the treatment means were assessed by Tukey's significance test at $P \leq 0.05$.

\section{Results and Discussion}

\subsection{Carotenoids}

Kale is considered to be an excellent source of various dietary carotenoids (Holden et al., 1999). According to the U.S. Department of Agriculture (USDA), kale has the highest concentration of lutein and $\beta$-carotene of any vegetable (USDA, 2016). At maturity, all three kale cultivars were comprised of $41.5-44.9 \%$ lutein, $21.6-23.9 \%$ $\beta$-carotene, $15.0-17.4 \%$ neoxanthin, $13.5-17.2 \%$ violaxanthin with the combination of antheraxanthin, $\alpha$-carotene and zeaxanthin, comprising $2.3-2.8 \%$ of total carotenoids. The range for lutein was $6.71-7.27 \mathrm{mg} / 100 \mathrm{~g} \mathrm{FW}$, while the range for $\beta$-carotene was 3.41-3.86 mg/100 g FW for all three cultivars at maturity (Table 1). These values were within ranges reported for 23 leafy B. oleracea cultivars grown in the field for lutein (4.8-13.4 $\mathrm{mg} / 100 \mathrm{~g} \mathrm{FW}$ ) and $\beta$-carotene (3.8-10 mg/100 g FW) (Kopsell, Kopsell, Lefsrud, Curran-Celentano, \& Dukach, 2004). However, $\beta$-carotene was lower than the range of 5.1-7.3 mg/100 g FW reported by Sikora and Bodziarczyk (2012) and the range of 6.1-11.6 mg/100 g FW reported by Lefsrud et al. (2007). Both studies evaluated 'Winterbor' kale, which differed from cultivars in the present study. Cultivation conditions also differed. Lefsrud et al. (2007) grew kale with full strength Hoagland nutrient in a hydroponic system. In our study, kale grown under identical conditions showed variability in carotenoid concentration depending on the cultivar. In general, lutein, $\beta$-carotene, and neoxanthin were higher in red leaf 'Scarlet' kale than in green leaf with purple vein 'RR' and green leaf 'DBC' kale at microgreen (MG1 and MG2) and BL2 stages.

Total carotenoid concentration increased as kale matured. The two major carotenoids, lutein and $\beta$-carotene, were significantly higher at the adult stage compared to microgreens in all kale cultivars (Table 1). Similarly, published studies reported higher $\beta$-carotene and lutein concentration in mature compared to younger kale (de Azevedo \& Rodriguez-Amaya, 2005; Lefsrud et al., 2007). Generally, individual and total carotenoids were higher at adult stage than microgreen stages in all three kale cultivars (Table 1), which agreed with reports that ripening in fruits and vegetables enhanced biosynthesis of carotenoids (Fraser, Truesdale, Bird, Schuch, \& Bramley, 1994). 
Table 1. Carotenoid concentration of three cultivars of fresh kale, Brassica oleracea L. 'Dwarf Blue Curled' (DBC), Brassica napus 'Red Russian' (RR), and Brassica oleracea L. 'Scarlet' (Scarlet), at five stages of leaf development

\begin{tabular}{|c|c|c|c|c|c|c|c|c|c|c|c|c|c|}
\hline \multirow{3}{*}{ Stage $^{z}$} & \multicolumn{4}{|c|}{ Lutein } & \multicolumn{3}{|c|}{$\beta$-carotene } & \multicolumn{3}{|c|}{ Neoxanthin } & \multicolumn{3}{|c|}{ Violaxanthin } \\
\hline & \multicolumn{4}{|c|}{ Cultivar } & \multicolumn{3}{|c|}{ Cultivar } & \multicolumn{3}{|c|}{ Cultivar } & \multicolumn{3}{|c|}{ Cultivar } \\
\hline & DBC & & RR & Scarlet & DBC & RR & Scarlet & DBC & RR & Scarlet & DBC & $\mathrm{RR}$ & Scarlet \\
\hline & \multicolumn{13}{|c|}{ 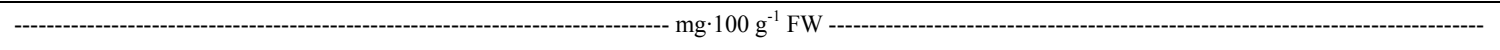 } \\
\hline MG1 & $3.63^{y}$ & de & $3.24 \mathrm{e}$ & 4.60 bcde & 1.83 ef & $2.05 \mathrm{def}$ & 2.66 bcdef & $1.09 \mathrm{~d}$ & $1.13 \mathrm{~d}$ & $1.68 \mathrm{bcd}$ & $0.47 \mathrm{f}$ & 0.79 ef & 0.75 ef \\
\hline MG2 & 3.43 & de & $2.90 \mathrm{e}$ & $4.29 \mathrm{cde}$ & $1.51 \mathrm{f}$ & 1.75 ef & $2.15 \mathrm{def}$ & $1.14 \mathrm{~d}$ & $0.99 \mathrm{~d}$ & $1.58 \mathrm{bcd}$ & $0.64 \mathrm{f}$ & 0.94 def & $1.10 \mathrm{cdef}$ \\
\hline BL1 & 7.13 & $a b$ & $4.81 \mathrm{abcd}$ & $5.90 \mathrm{abcd}$ & $4.10 \mathrm{a}$ & 3.18 abcde & $3.33 \mathrm{abcd}$ & $2.57 \mathrm{ab}$ & $1.75 \mathrm{abcd}$ & $2.17 \mathrm{abc}$ & 1.58 bcde & $1.80 \mathrm{bcd}$ & $1.98 \mathrm{abc}$ \\
\hline BL2 & 4.57 & bcde & $3.81 \mathrm{de}$ & $6.46 \mathrm{abc}$ & $2.30 \mathrm{cdef}$ & $2.22 \mathrm{def}$ & $3.46 \mathrm{abcd}$ & $1.36 \mathrm{~cd}$ & $1.23 \mathrm{~cd}$ & $2.41 \mathrm{ab}$ & $1.75 \mathrm{bcd}$ & 1.56 bcde & $2.22 \mathrm{ab}$ \\
\hline Adult & 7.27 & $\mathrm{a}$ & $6.71 \mathrm{abc}$ & $7.08 \mathrm{ab}$ & $3.73 \mathrm{abc}$ & $3.86 \mathrm{ab}$ & $3.41 \mathrm{abcd}$ & $2.57 \mathrm{ab}$ & $2.43 \mathrm{ab}$ & $2.74 \mathrm{a}$ & $2.26 \mathrm{ab}$ & $2.77 \quad \mathrm{a}$ & $2.13 \mathrm{ab}$ \\
\hline $\mathrm{SE}^{\mathrm{x}}$ & \multicolumn{4}{|c|}{0.51} & \multicolumn{3}{|l|}{0.28} & \multicolumn{3}{|l|}{0.20} & \multicolumn{3}{|l|}{0.17} \\
\hline \multicolumn{2}{|c|}{ Cultivar (C) } & \multicolumn{3}{|c|}{0.0005} & \multicolumn{3}{|l|}{0.0854} & \multicolumn{3}{|l|}{$\leq 0.0001$} & \multicolumn{3}{|l|}{0.0272} \\
\hline \multicolumn{2}{|c|}{ Stage (S) } & \multicolumn{3}{|c|}{$\leq 0.0001$} & \multicolumn{3}{|l|}{$\leq 0.0001$} & \multicolumn{3}{|l|}{$\leq 0.0001$} & \multicolumn{3}{|l|}{$\leq 0.0001$} \\
\hline \multirow[t]{2}{*}{$\mathrm{C} \times \mathrm{S}$} & \multicolumn{4}{|c|}{0.1175} & \multicolumn{3}{|l|}{0.0179} & \multicolumn{3}{|l|}{0.0424} & \multicolumn{3}{|l|}{0.0826} \\
\hline & & & ntheraxanthi & & & $\alpha$-carotene & & & Zeaxanthin & & & tal carotenoid & \\
\hline Stage & & & Cultivar & & & Cultivar & & & Cultivar & & & Cultivar & \\
\hline & $\mathrm{DBC}$ & & $\mathrm{RR}$ & Scarlet & $\mathrm{DBC}$ & $\mathrm{RR}$ & Scarlet & $\mathrm{DBC}$ & $\mathrm{RR}$ & Scarlet & $\mathrm{DBC}$ & $\mathrm{RR}$ & Scarlet \\
\hline & ---- & . & 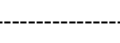 & - & - & 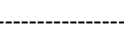 & mg & $\mathrm{g}^{-1} \mathrm{FW}$ & - & . & - & - & - \\
\hline MG1 & 0.14 & cde & 0.14 cde & 0.13 cde & $0.09 \mathrm{e}$ & $0.10 \mathrm{e}$ & $0.12 \mathrm{e}$ & $0.02 \mathrm{f}$ & $0.03 \mathrm{f}$ & $0.05 \mathrm{def}$ & $7.28 \mathrm{e}$ & $7.49 \mathrm{e}$ & 9.99 bcde \\
\hline MG2 & 0.13 & de & $0.09 \mathrm{e}$ & $0.09 \mathrm{e}$ & $0.10 \mathrm{e}$ & $0.09 \mathrm{e}$ & 0.13 bcde & $0.04 \mathrm{ef}$ & $0.06 \mathrm{def}$ & $0.10 \mathrm{bcd}$ & $7.00 \mathrm{e}$ & $6.81 \mathrm{e}$ & 9.46 cde \\
\hline BL1 & 0.39 & $\mathrm{a}$ & $0.26 \mathrm{abc}$ & $0.24 \mathrm{bcd}$ & $0.19 \mathrm{abcd}$ & $0.13 \mathrm{cde}$ & $0.21 \mathrm{ab}$ & $0.19 \mathrm{a}$ & 0.10 bcde & $0.12 \mathrm{bc}$ & $16.15 \mathrm{a}$ & 12.03 abcde & $13.94 \mathrm{abcd}$ \\
\hline BL2 & 0.17 & cde & $0.10 \mathrm{e}$ & $0.35 \mathrm{ab}$ & 0.14 bcde & $0.13 \mathrm{de}$ & $0.20 \mathrm{abcd}$ & $0.07 \mathrm{cdef}$ & $0.05 \mathrm{ef}$ & $0.13 \mathrm{ab}$ & 10.35 abcde & $9.09 \mathrm{de}$ & $15.23 \mathrm{abc}$ \\
\hline Adult & 0.16 & cde & $0.11 \mathrm{de}$ & $0.17 \mathrm{cde}$ & $0.24 \mathrm{a}$ & $0.23 \mathrm{a}$ & $0.21 \mathrm{abc}$ & $0.06 \mathrm{def}$ & $0.04 \mathrm{f}$ & 0.05 ef & $16.28 \mathrm{a}$ & $16.15 \mathrm{a}$ & $15.78 \mathrm{ab}$ \\
\hline $\mathrm{SE}$ & & 0.03 & & & 0.01 & & & 0.01 & & & 1.17 & & \\
\hline Cultiva & $\operatorname{ar}(C)$ & 0.00 & & & 0.0015 & & & $\leq 0.0001$ & & & 0.0050 & & \\
\hline Stage ( & & $\leq 0$ & 001 & & $\leq 0.0001$ & & & $\leq 0.0001$ & & & $\leq 0.0001$ & & \\
\hline $\mathrm{C} \times \mathrm{S}$ & & $\leq 0$ & 001 & & 0.0113 & & & $\leq 0.0001$ & & & 0.0698 & & \\
\hline
\end{tabular}

Note. ${ }^{\mathrm{z}} \mathrm{MG1}$, kale with two fully expanded cotyledons (microgreen 1); MG2, kale with two fully expanded true leaves (microgreen 2); BL1, kale with four fully expanded true leaves (baby leaf 1); BL2, kale with six fully expanded true leaves (baby leaf 2); Adult, kale with more than eight fully expanded true leaves.

${ }^{\mathrm{y}}$ Values are the means of four replications $(\mathrm{n}=4)$.

${ }^{x}$ Pooled standard error.

${ }^{\mathrm{w}}$ The total carotenoids were the sum of concentrations of individual carotenoids.

Different lower case letters indicate significant difference of each caroteniod by Tukey's significance test at $P \leq$ 0.05 .

Interestingly, upon maturity there were no significant differences in individual carotenoids and total carotenoids among kale cultivars (Table 1). There was no interaction between cultivars and developmental stages in total carotenoid, although the main effects, cultivar and developmental stage, showed significant differences. In addition to carotenoids, kale is high in antioxidant phenolic compounds, which may further contribute to kale having the highest antioxidant capacity among Brassica vegetables (Podsędek, 2007; Olsen et al., 2010).

\subsection{Total Phenolics and Anthocyanins}

Biosynthesis of phenolic compounds contribute to plant photosynthesis, growth, and development (Crozier, Jaganath, \& Clifford, 2006). In addition, consumption of plants rich in phenolic compounds provide various health benefits to humans. In the current study, total phenolic concentration was significantly higher in red leaf 'Scarlet' than green leaf with purple veins ' $R R$ ' and green leaf ' $D B C$ ' kale, but there was no significant difference between 'DBC' and 'RR' (Table 2). Studies have reported higher total phenolic content in red compared to green cultivars of lettuce (Lactuca sativa L.) (Liu et al., 2007; Llorach, Martínez-Sánchez, Tomás-Barberán, Gil, \& Ferreres, 2008; Kim et al., 2016). Regardless of leaf color, total phenolics in all three kale cultivars were higher in BL2 and adult stages (128.3-250.5 mg GAE/100 g FW) than early stages (MG1, MG2 and BL1, 54.6-170.1.0 mg GAE/100 g FW) (Table 2). Total phenolic concentration in our study was within 
the range of 145-284 mg/100 g FW reported for six different kale genotypes (Armesto, Carballo, \& Martínez, 2015). Total phenolic range was lower than the range of 322.3-390.0 mg GAE/100 g FW reported for 'Galega' kale likely due to differences in cultivars, growing environment and the extraction method for total phenolics (Armesto et al., 2015).

The present study also evaluated anthocyanin content since this subgroup of phenolic compound is the main plant pigment responsible for the red pigmentation in plants (Ferreres, Gil, Castaner, \& Tomás-Barberán, 1997). As expected anthocyanin concentration was low in green leaf 'DBC' kale with red leaf 'Scarlet' kale having higher anthocyanin concentration at all developmental stages (Table 2). Red leaf 'Scarlet' kale also contained significantly higher level of anthocyanins than green leaf with purple veins 'RR' kale at all developmental stages. In red leaf 'Scarlet' kale, anthocyanins were influenced by developmental stage with higher concentration in BL2 and adult stage compared to younger microgreen and BL1 stages.

Table 2. Total phenolic compounds and anthocyanin concentrations of three cultivars of fresh kale, Brassica oleracea L. 'Dwarf Blue Curled' (DBC), Brassica napus 'Red Russian' (RR), and Brassica oleracea L. 'Scarlet' (Scarlet), at five developmental stages

\begin{tabular}{|c|c|c|c|c|c|c|c|c|c|c|}
\hline \multirow{3}{*}{ Stage $^{\mathrm{z}}$} & \multicolumn{5}{|c|}{ Total phenolic } & \multicolumn{5}{|c|}{ Total anthocyanin } \\
\hline & \multicolumn{5}{|c|}{ Cultivar } & \multicolumn{5}{|c|}{ Cultivar } \\
\hline & $\mathrm{DBC}$ & RR & & Scarlet & & $\overline{\mathrm{DBC}}$ & RR & & Scarle & \\
\hline & \multicolumn{10}{|c|}{ 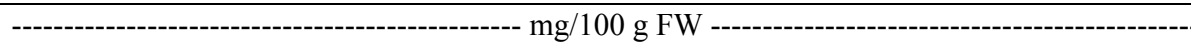 } \\
\hline MG1 & $61.7^{\mathrm{y}} \mathrm{g}$ & 54.6 & $\mathrm{~g}$ & 85.2 & fg & $0.1 \mathrm{~h}$ & 8.6 & efg & 12.6 & de \\
\hline MG2 & $64.3 \mathrm{~g}$ & 60.3 & $\mathrm{~g}$ & 113.0 & ef & $0.0 \mathrm{~h}$ & 9.4 & ef & 16.7 & $\mathrm{~d}$ \\
\hline BL1 & $73.2 \mathrm{~g}$ & 69.4 & $\mathrm{~g}$ & 170.1 & $\mathrm{~b}$ & $0.2 \mathrm{~h}$ & 2.2 & fgh & 24.6 & $\mathrm{c}$ \\
\hline BL2 & $161.3 \mathrm{bc}$ & 156.5 & bcd & 245.2 & $\mathrm{a}$ & $1.0 \mathrm{~h}$ & 8.5 & efg & 40.9 & $\mathrm{~b}$ \\
\hline Adult & $128.3 \mathrm{de}$ & 133.6 & cde & 250.5 & $\mathrm{a}$ & $1.4 \mathrm{gh}$ & 7.2 & efgh & 48.4 & $\mathrm{a}$ \\
\hline $\mathrm{SE}^{\mathrm{W}}$ & \multicolumn{5}{|l|}{6.4} & \multicolumn{5}{|l|}{1.4} \\
\hline Cultivar (C) & \multicolumn{5}{|l|}{$\leq 0.0001$} & \multicolumn{5}{|l|}{$\leq 0.0001$} \\
\hline Stage (S) & \multicolumn{5}{|l|}{$\leq 0.0001$} & \multicolumn{5}{|l|}{$\leq 0.0001$} \\
\hline $\mathrm{C} \times \mathrm{S}$ & \multicolumn{5}{|l|}{$\leq 0.0001$} & \multicolumn{5}{|l|}{$\leq 0.0001$} \\
\hline
\end{tabular}

Note. ${ }^{\mathrm{Z} M G 1}$, kale with two fully expanded cotyledons (microgreen 1); MG2, kale with two fully expanded true leaves (microgreen 2); BL1, kale with four fully expanded true leaves (baby leaf 1); BL2, kale with six fully expanded true leaves (baby leaf 2); Adult, kale with more than eight fully expanded true leaves.

${ }^{\mathrm{y}}$ Values are the means of four replications $(\mathrm{n}=4)$.

${ }^{\mathrm{w}}$ Pooled standard error.

Different lower case letters indicate significant difference of total phenolic or anthocyanin by Tukey's significance test at $P \leq 0.05$.

There was an interaction $(P \leq 0.001)$ between cultivars and developmental stages for both total phenolic compounds and anthocyanins (Table 2). Based on the study results, mature red leaf 'Scarlet' kale had the highest total phenolics and anthocyanin concentrations.

In all three cultivars, higher carotenoid, total phenolics, and anthocyanin concentrations were detected in fresh BL2 or adult stage than younger fresh microgreen stages. However, results differed depending on calculation on a FW versus DW basis. Calculated as DW resulted in the opposite effect of higher carotenoid concentration in microgreen than BL2 and adult leaves in all three kales (Table 3). Total phenolic concentration was higher in 'DBC' and 'RR' kale microgreens (MG1 and MG2, 11.0-12.4 mg GAE ${ }^{-1} \mathrm{DW}$ ) than in baby and adult leaves (6.6-9.9 $\mathrm{mg} \mathrm{GAE} \mathrm{g}^{-1} \mathrm{DW}$ ). Anthocyanins calculated as DW, 'RR' kale also had the opposite results to fresh leaves of higher total anthocyanin concentration at the earlier stage of leaf development (microgreens) and decreased as leaves matured. Both phytochemical accumulation difference and dry mass at different leaf development stage may explain discrepancies in results when expressed as DW versus FW. Dry mass increased with maturation of kale. The dry mass range for kale microgreens was $4.9-8.4 \%$ and $17.2-20.1 \%$ at the adult stage (Figure 1). Similarly, previous studies reported dry mass of adult kale to be between 17.1-23.9\% (Sikora \& Bodziarczyk, 2012). Overall, this resulted in a dry mass increase from microgreen stage to adult stage of 4.1 times in 'RR', 3.5 times in 'DBC', and 3 times in 'Scarlet' kale. 
Table 3. Total carotenoids, phenolic compounds and anthocyanin concentrations based upon dry weight of three cultivars of kale, Brassica oleracea L. 'Dwarf Blue Curled' (DBC), Brassica napus 'Red Russian' (RR), and Brassica oleracea L. 'Scarlet' (Scarlet), at five stages of leaf development

\begin{tabular}{|c|c|c|c|c|c|c|c|c|c|c|c|c|}
\hline \multirow{3}{*}{ Stage $^{z}$} & \multicolumn{5}{|c|}{ Total carotenoid $^{\mathrm{y}}$} & \multicolumn{3}{|c|}{ Total phenolic compounds } & \multicolumn{4}{|c|}{ Total anthocyanin } \\
\hline & \multicolumn{5}{|c|}{ Cultivar } & \multicolumn{3}{|c|}{ Cultivar } & \multicolumn{4}{|c|}{ Cultivar } \\
\hline & $\mathrm{DBC}$ & & $\mathrm{RR}$ & & Scarlet & $\mathrm{DBC}$ & $\mathrm{RR}$ & Scarlet & $\mathrm{DBC}$ & $\mathrm{RR}$ & & Scarlet \\
\hline & \multicolumn{5}{|c|}{ 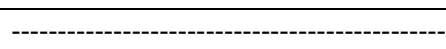 } & - & $\mathrm{mg} \cdot \mathrm{g}^{-1} \mathrm{DV}$ & & 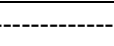 & 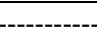 & & --------.. \\
\hline MG1 & $1.5^{\mathrm{x}}$ & bcde & 1.5 & abcd & $1.6 \mathrm{abc}$ & $12.4 \mathrm{bc}$ & $11.2 \mathrm{~cd}$ & $13.6 \mathrm{ab}$ & $14.0 \mathrm{~d}$ & 1736.5 & $\mathrm{c}$ & $2013.0 \mathrm{bc}$ \\
\hline MG2 & 1.2 & defg & 1.3 & cdef & $1.1 \mathrm{efgh}$ & $11.0 \mathrm{~cd}$ & $11.2 \mathrm{~cd}$ & $13.5 \mathrm{ab}$ & $0.0 \mathrm{~d}$ & 1740.2 & $\mathrm{c}$ & $1987.7 \mathrm{bc}$ \\
\hline BL1 & 1.8 & $\mathrm{a}$ & 1.6 & $\mathrm{ab}$ & $1.1 \mathrm{efgh}$ & 8.2 & 9.4 ef & $13.7 \mathrm{ab}$ & $22.7 \mathrm{~d}$ & 290.4 & $\mathrm{~d}$ & $1971.9 \mathrm{bc}$ \\
\hline BL2 & 0.6 & $\mathrm{ij}$ & 0.6 & $\mathrm{j}$ & 0.9 fghij & 9.3 & 9.9 de & $14.9 \mathrm{a}$ & $58.9 \mathrm{~d}$ & 534.9 & $\mathrm{~d}$ & $2494.7 \mathrm{ab}$ \\
\hline Adult & 0.9 & fghi & 0.8 & hij & 0.8 ghij & 7.5 & $6.6 \mathrm{~h}$ & $13.4 \mathrm{ab}$ & $84.4 \mathrm{~d}$ & 363.5 & $\mathrm{~d}$ & $2968.1 \mathrm{a}$ \\
\hline $\mathrm{SE}^{\mathrm{w}}$ & \multicolumn{5}{|l|}{0.1} & \multicolumn{3}{|l|}{0.3} & \multicolumn{4}{|l|}{120.6} \\
\hline Cultivar (C) & \multicolumn{5}{|c|}{0.1758} & \multicolumn{3}{|l|}{$\leq 0.0001$} & \multicolumn{4}{|l|}{$\leq 0.0001$} \\
\hline Stage (S) & \multicolumn{5}{|c|}{$\leq 0.0001$} & \multicolumn{3}{|l|}{$\leq 0.0001$} & \multicolumn{4}{|l|}{$\leq 0.0001$} \\
\hline $\mathrm{C} \times \mathrm{S}$ & \multicolumn{5}{|c|}{$\leq 0.0001$} & \multicolumn{3}{|l|}{$\leq 0.0001$} & \multicolumn{4}{|l|}{$\leq 0.0001$} \\
\hline
\end{tabular}

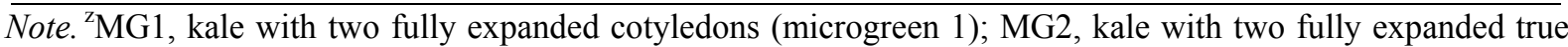
leaves (microgreen 2); BL1, kale with four fully expanded true leaves (baby leaf 1); BL2, kale with six fully expanded true leaves (baby leaf 2); Adult, kale with more than eight fully expanded true leaves.

${ }^{\mathrm{y}}$ The total carotenoids were the sum of concentrations of individual carotenoids.

${ }^{\mathrm{x}}$ Values are the means of four replications $(\mathrm{n}=4)$.

${ }^{\mathrm{w}}$ Pooled standard error.

Different lower case letters indicate significant difference of total carotenoid, phenolic compounds or anthocyanin by Tukey's significance test at $P \leq 0.05$.

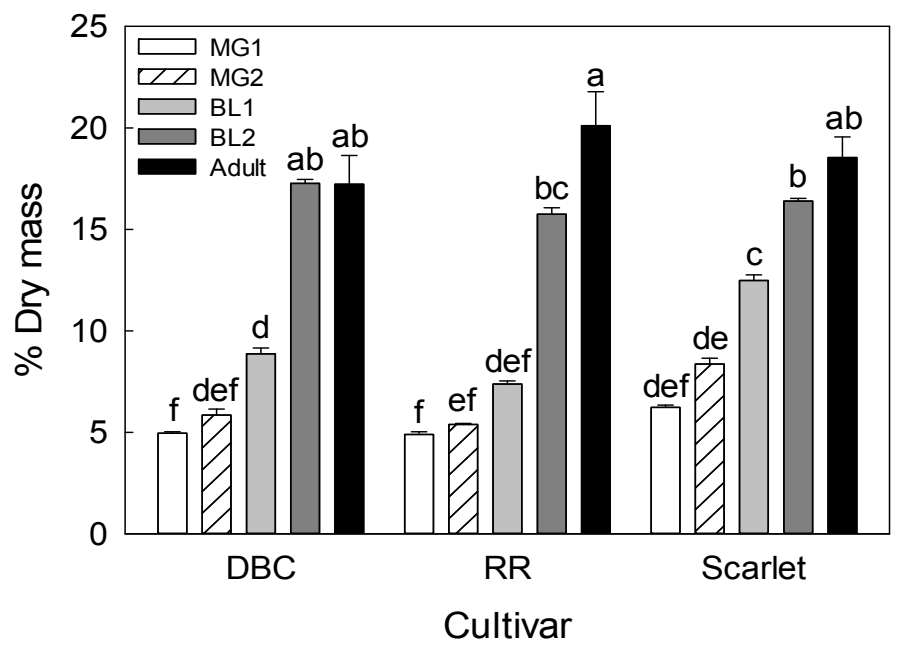

Figure 1. Percent dry mass of three cultivars of kale leaves at five stages of leaf development (Brassica oleracea L. 'Dwarf Blue Curled', Brassica napus 'Red Russian' and Brassica oleracea L. 'Scarlet'). MG1; kale with two fully expanded cotyledons (microgreen 1); MG2; kale with two fully expanded true leaves (microgreen 2); BL1; kale with four fully expanded true leaves (baby leaf 1); BL2; kale with six fully expanded true leaves (baby leaf 2); Adult; kale with more than eight fully expanded true leaves. 'DBC'; green leaf kale; 'RR'; green leaf with purple veins kale and 'Scarlet'; red leaf kale. Values are the means and standard errors of four replications ( $\mathrm{n}=$

4). Mean separation among the developmental stages within each cultivar by

Tukey's significance test at $P \leq 0.05$

\section{Conclusions}

Cultivar influenced carotenoid, phenolic, and anthocyanin concentration in kale. Overall, 'Scarlet' kale was the highest in total carotenoids, phenolics, and anthocyanins while 'DBC' and 'RR' kale were generally similar for 
total carotenoids and phenolic compounds. Red leaf 'Scarlet' and green leaf 'DBC' are same species (B. oleracea L.), while green leaf with purple veins 'RR' belongs to a different kale species (B. napus), indicating that leaf color is important. Carotenoids and phenolic compounds are major sources of plant pigmentation. Developmental stage also influenced carotenoid, phenolic, and anthocyanin concentrations in kale. Overall, carotenoids, phenolics, and anthocynanins increased as kale matured. Increase in these antioxidants coincided with increased dry mass during leaf development. On a DW basis, total carotenoids were more abundant in microgreens and baby greens than adult stage. In contrast, on a FW basis, baby greens or adult kales generally contained more carotenoids and total phenolic compounds than microgreens. This discrepancy could be more likely due to the difference of dry mass and phytochemical accumulation at different leaf development stages. Although microgreens may be more nutritious for some vegetables, based on our study mature plants contained more health-beneficial bioactive compounds with antioxidant properties. Leaf color was also an influencing factor in kale nutritional value. Although increasing carotenoid biosynthesis during maturation resulted in no differences in carotenoid concentration between adult red and green leaf kale, the highest concentration of phenolic compounds was provided by adult red leaf 'Scarlet' kale.

\section{Reference}

Armesto, J., Carballo, J., \& Martínez, S. (2015). Physicochemical and phytochemical properties of two phenotypes of galega kale (Brassica oleracea L. var. Acephala cv. Galega). Journal of Food Biochemistry, 39, 439-448. https://doi.org/10.1111/jfbc.12151

Bartley, G. E., \& Scolnik, P. A. (1995). Plant carotenoids: Pigments for photoprotection, visual attraction, and human health. The Plant Cell, 7, 1027-1038. https://doi.org/10.1105/tpc.7.7.1027

Crozier, A., Jaganath, L. B., \& Clifford, M. N. (2006). Plant secondary metabolites: Occurrence, structure and role in the human diet. Oxford, UK: Blackwell Publishing.

de Azevedo, C. H., \& Rodriguez-Amaya, D. B. (2005). Carotenoid composition of kale as influenced by maturity, season and minimal processing. Journal of the Science of Food Agriculture, 85, 591-597. https://doi.org/10.1002/jsfa.1993

Ferreres, F., Gil, M. I., Castaner, M., \& Tomás-Barberán, F. A. (1997). Phenolic metabolites in red pigmented lettuce (Lactuca sativa). Changes with minimal processing and cold storage. Journal of Agricultural and Food Chemistry, 45, 4249-4254. https://doi.org/10.1021/jf970399j

Fraser, P. D., Truesdale, M. R., Bird, C. R., Schuch, W., \& Bramley, P. M. (1994). Carotenoid biosynthesis during tomato fruit development (evidence for tissue-specific gene expression). Plant Physiology, 105, 405-413.

He, J., \& Giusti, M. M. (2010). Anthocyanins: Natural colorants with health-promoting properties. Annual Review of Food Science and Technology, 1, 163-187. https://doi.org/10.1104/pp.105.1.405

Holden, J. M., Eldridge, A. L., Beecher, G. R., Buzzard, I. M., Bhagwat, S., Davis, C. S., ... Schakel, S. (1999). Carotenoid content of U.S. foods: An update of the database. Journal of Food Composition Analysis, 12, 169-196.

Ismail, A., Marjan, Z. M., \& Foong, C. W. (2004). Total antioxidant activity and phenolic content in selected vegetables. Food Chemistry, 87, 581-586. https://doi.org/10.1016/j.foodchem.2004.01.010

Kapusta-Duch, J., Kopec, A., Piatkowska, E., Borczak, B., \& Leszczynska, T. (2012). The beneficial effects of Brassica vegetables on human health. Roczniki Państwowego Zakladu Higieny, 63, 389-395.

Kim, M. J., Moon, Y., Kopsell, D. A., Park, S., Tou, J. C., \& Waterland, N. L. (2016). Nutritional value of crisphead 'Iceberg' and romaine lettuces (Lactuca sativa L.). Journal of Agricultural Science, 8(11), 1-10.

Kopsell, D. A., Barickman, T. C., Sams, C. E., \& McElroy, J. S. (2007). Influence of nitrogen and sulfur on biomass production and carotenoid and glucosinolate concentrations in watercress (Nasturtium officinale R. Br.). Journal of Agricultural and Food Chemistry, 55, 10628-10634. https://doi.org/10.1021/jf072793f

Kopsell, D. A., Kopsell, D. E., Lefsrud, M. G., Curran-Celentano, J., \& Dukach, L. E. (2004). Variation in lutein, $\beta$-carotene, and chlorophyll concentrations among Brassica oleracea cultigens and seasons. HortScience, 39, 361-364.

Lefsrud, M., Kopsell, D., Wenzel, A., \& Sheehan, J. (2007). Changes in kale (Brassica oleracea L. var. acephala) carotenoid and chlorophyll pigment concentrations during leaf ontogeny. Scientia Horticulturae, 112, 136-141. https://doi.org/10.1016/j.scienta.2006.12.026 
Liu, X., Ardo, S., Bunning, M., Parry, J., Zhou, K., Stushnoff, C., ... Kendall, P. (2007). Total phenolic content and DPPH radical scavenging activity of lettuce (Lactuca sativa L.) grown in Colorado. LWT - Food Science and Technology, 40, 552-557. https://doi.org/10.1016/j.lwt.2005.09.007

Llorach, R., Martínez-Sánchez, A., Tomás-Barberán, F. A., Gil, M. I., \& Ferreres, F. (2008). Characterisation of polyphenols and antioxidant properties of five lettuce varieties and escarole. Food Chemistry, 108, 1028-1038. https://doi.org/10.1016/j.foodchem.2007.11.032

Nicolle, C., Carnat, A., Fraisse, D., Lamaison, J. L., Rock, E., Michel, H., ... Remesy, C. (2004). Characterisation and variation of antioxidant micronutrients in lettuce (Lactuca sativa folium). Journal of the Science of Food Agriculture, 84, 2061-2069. https://doi.org/10.1002/jsfa.1916

Olsen, H., Aaby, K., \& Borge, G. I. A. (2010). Characterization, quantification, and yearly variation of the naturally occurring polyphenols in a common red variety of curly kale (Brassica oleracea L. convar. acephala var. sabellica cv.'Redbor'). Journal of Agricultural and Food Chemistry, 58, 11346-11354. https://doi.org/10.1021/jf102131g

Pagliaro, B., Santolamazza, C., Simonelli, F., \& Rubattu, S. (2015). Phytochemical compounds and protection from cardiovascular diseases: A state of the art. BioMed Research International, 2015. https://doi.org/ $10.1155 / 2015 / 918069$

Perry, A., Rasmussen, H., \& Johnson, E. (2009). Xanthophyll (lutein, zeaxanthin) content in fruits, vegetables and corn and egg products. Journal of Food Composition Analysis, 22, 9-15. https://doi.org/10.1016/ j.jfca.2008.07.006

Podsędek, A. (2007). Natural antioxidants and antioxidant capacity of Brassica vegetables: A review. LWT-Food Science and Technology, 40, 1-11. https://doi.org/10.1016/j.lwt.2005.07.023

Rodríguez-Cantú, L. N., Gutiérrez-Uribe, J. A., Arriola-Vucovich, J., Díaz-De La Garza, R. I., Fahey, J. W., \& Serna-Saldivar, S. O. (2011). Broccoli (Brassica oleracea var. italica) sprouts and extracts rich in glucosinolates and isothiocyanates affect cholesterol metabolism and genes involved in lipid homeostasis in hamsters. Journal of Agricultural and Food Chemistry, 59, 1095-1103. https://doi.org/10.1021/jf103513w

Sikora, E., \& Bodziarczyk, I. (2012). Composition and antioxidant activity of kale (Brassica oleracea L. var. acephala) raw and cooked. Acta Scientiarum Polonorum. Technologia Alimentaria, 11, 239-248.

Singh, J., Upadhyay, A., Prasad, K., Bahadur, A., \& Rai, M. (2007). Variability of carotenes, vitamin C, E and phenolics in Brassica vegetables. Journal of Food Composition Analysis, 20, 106-112. https://doi.org/ 10.1016/j.jfca.2006.08.002

U.S. Department of Agriculture. (2016). National nutrient database for standard reference, release 28. Washington, D.C., USA: U.S. Department of Agriculture.

Walsh, R. P., Bartlett, H., \& Eperjesi, F. (2015). Variation in carotenoid content of kale and other vegetables: A review of pre-and post-harvest effects. Journal of Agricultural Food Chemistry, 63, 9677-9682. https://doi.org/10.1021/acs.jafc.5b03691

Wu, Q. J., Yang, Y., Wang, J., Han, L. H., \& Xiang, Y. B. (2013). Cruciferous vegetable consumption and gastric cancer risk: A meta-analysis of epidemiological studies. Cancer Science, 104, 1067-1073. https://doi.org/10.1111/cas.12195

Xiao, Z., Lester, G. E., Luo, Y., \& Wang, Q. (2012). Assessment of vitamin and carotenoid concentrations of emerging food products: Edible microgreens. Journal of Agricultural Food Chemistry, 60, 7644-7651. https://doi.org/10.1021/jf300459b

\section{Copyrights}

Copyright for this article is retained by the author(s), with first publication rights granted to the journal.

This is an open-access article distributed under the terms and conditions of the Creative Commons Attribution license (http://creativecommons.org/licenses/by/4.0/). 